\title{
Torsion-free groups with indecomposable holonomy group. I
}

\author{
V. A. Bovdi, P. M. Gudivok and V. P. Rudko \\ (Communicated by S. Sidki) \\ Dedicated to Professor L.G. Kovács on his 65th birthday
}

\begin{abstract}
We study the torsion-free generalized crystallographic groups with indecomposable holonomy group which is isomorphic to either $C_{p^{s}}$ or $C_{p} \times C_{p}$.
\end{abstract}

\section{Introduction}

A classical crystallographic group is a discrete cocompact subgroup of $I\left(\mathbb{R}^{m}\right)$, the isometry group of $\mathbb{R}^{m}$. Torsion-free crystallographic groups are called Bieberbach groups. The present state of the theory of crystallographic groups and a historical overview, as well as its connections to other branches of mathematics, are described in $[17,18]$.

In this paper we consider generalized torsion-free crystallographic groups with indecomposable holonomy groups isomorphic to either $C_{p^{s}}$ or $C_{p} \times C_{p}$.

It was shown in $[7,8,14]$ that the description of the $n$-dimensional crystallographic groups for arbitrary $n$ is of wild type, in the sense that it is related to the classical unsolvable problem of describing the canonical forms of pairs of linear operators on finite-dimensional vector spaces.

Using Diederichsen's classification of integral representations of cyclic groups of prime order (see [6]), Charlap [5] gave a full classification of Bieberbach groups with cyclic holonomy group $G$ of prime order. Hiss and Szczepánski [13] proved that there are no Bieberbach groups with non-trivial irreducible holonomy group. Kopcha and Rudko [14] studied torsion-free crystallographic groups with indecomposable cyclic holonomy group of order $p^{n}$, the classification of which for $n \geqslant 5$ also has wild type.

Cobb [5] constructed an infinite family of compact flat manifolds with first Betti number zero and holonomy group isomorphic to $C_{2} \times C_{2}$. In $[19,20,21]$ Rossetti

This research was supported by OTKA No. T 025029 and T 037202 
and Tirao described the torsion-free crystallographic groups whose holonomy groups are direct sums of indecomposable subgroups of $\operatorname{GL}(n, \mathbb{Z})(n \leqslant 5)$ and isomorphic to $C_{2} \times C_{2}$.

Further interesting results on this topic were obtained in the research of Gupta and Sidki $[9,10]$.

We need the following definitions and notation for the statement of our results.

Let $K$ be a principal domain, let $F$ be a field containing $K$ and let $G$ be a finite group. Let $M$ be a $K G$-module of a faithful matrix $K$-representation $\Gamma$ of $G$ and let $F M$ be a vector space over $F$ in which $M$ is a full lattice. Let $\hat{M}=F M^{+} / M^{+}$be the quotient group of the additive group $F M^{+}$of $F M$ by the additive group $M^{+}$of $M$. Then $F M$ is an $F G$-module and $\hat{M}$ is a $K G$-module with operations defined by

$$
g \cdot(\alpha m)=\alpha g(m), \quad g \cdot(x+M)=g(x)+M,
$$

for $g \in G, \alpha \in F, m \in M, x \in F M$.

Let $T: G \rightarrow \hat{M}$ be a 1 -cocycle of $G$ with values in $\hat{M}$; thus each $T(g)$ is a coset of the form $x+M$. We define the group

$$
\mathfrak{C r y s}(G ; M ; T)=\{(g, x) \mid g \in G, x \in T(g)\}
$$

with the operation

$$
(g, x) \cdot\left(g^{\prime}, x^{\prime}\right)=\left(g g^{\prime}, g^{\prime} x+x^{\prime}\right),
$$

for $g, g^{\prime} \in G, x \in T(g), x^{\prime} \in T\left(g^{\prime}\right)$.

The purpose of this paper is to study the group $\operatorname{Crns}(G ; M ; T)$, and in particular to determine when it is torsion-free. We note that if $K=\mathbb{Z}$ and $F=\mathbb{R}$ then $\mathfrak{C} \mathfrak{x y}(G ; M ; T)$ is isomorphic to an $n$-dimensional classical crystallographic group, where $n=\operatorname{rank}_{\mathbb{Z}} M$.

We use the terminology of the theory of group representations. The group $\operatorname{Crys}(G ; M ; T)$ is called irreducible (resp. indecomposable) if $M$ is an irreducible (resp. indecomposable) $K G$-module and the cocycle $T$ is not cohomologous to zero.

A cocycle $T: G \rightarrow \hat{M}$ is called a coboundary if there exists an $x \in F M$ such that $T(g)=(g-1) x+M$ for every $g \in G$. Cocycles $T_{1}: G \rightarrow \hat{M}$ and $T_{2}: G \rightarrow \hat{M}$ are called cohomologous if $T_{1}-T_{2}$ is a coboundary.

Let $C^{1}(G, \hat{M}), B^{1}(G, \hat{M})$ and $H^{1}(G, \hat{M})=C^{1}(G, \hat{M}) / B^{1}(G, \hat{M})$ be respectively the group of cocycles, the group of coboundaries and the cohomology group of $G$ with values in $\hat{M}$. The group $\operatorname{Cry} \mathfrak{s}(G ; M ; T)$ is an extension of $M^{+}$by $G$; the extension splits if and only if $T \in B^{1}(G, \hat{M})$. Therefore $\operatorname{Crys}(G ; M ; T)$ splits for all $T$ if and only if $H^{1}(G, \hat{M})$ is trivial.

Throughout the paper, we write $\mathbb{Z}, \mathbb{Z}_{(p)}$ and $\mathbb{Z}_{p}$ respectively for the ring of rational integers, the localization of $\mathbb{Z}$ at the prime $p$ and the ring of $p$-adic integers.

\section{Main results}

Using results from $[2,3,11,12,15]$, we prove the following three theorems. Lemma 12 is also of independent interest. 
Theorem 1. Let $K$ be one of the rings $\mathbb{Z}, \mathbb{Z}_{p}, \mathbb{Z}_{(p)}$ and let $G \cong C_{p^{s}}$ be a cyclic group of order $p^{s}$. If $s \geqslant 3$, then the set of $K$-dimensions of the indecomposable $K_{p^{s}}$-modules $M$ for which there exist torsion-free groups $\mathbf{C r y s}\left(C_{p^{s}} ; M ; T\right)$, is unbounded.

Theorem 2. Let $K$ be $\mathbb{Z}_{(p)}$ or $\mathbb{Z}_{p}$ and let $G=\langle a\rangle \cong C_{p^{2}}$. Up to isomorphism, all torsionfree indecomposable groups $\operatorname{Crys}\left(C_{p^{2}} ; M ; T\right)$ are described in terms of the following indecomposable $K_{p^{2}}$-modules $M$ and cocycles $T$ of $C_{p^{2}}$ with values in the groups $\hat{M}=$ $F M^{+} / M^{+}$:

(1) $M=X_{i}=\left\langle(a-1) \Phi\left(a^{p}\right), \Phi(a)+(a-1)^{i+1}\right\rangle$ and $T=T_{i}$, where

$$
\Phi(x)=x^{p-1}+\cdots+x+1, \quad T_{i}(a)=p^{-2} \Phi(a) \Phi\left(a^{p}\right)+X_{i},
$$

for $i=0,1, \ldots, p-2$;

(2) $M=U_{j}=\left\langle\left((a-1)^{j+1}+\Phi(a),(a-1)^{j}\right), \Phi\left(a^{p}\right)(a-1,1)\right\rangle$, a KC $C_{p^{2}}$-submodule of $\left(K C_{p^{2}}\right)^{(2)}=\left\{\left(x_{1}, x_{2}\right) \mid x_{1}, x_{2} \in K C_{p^{2}}\right\}$, and $T=f_{j}$, where

$$
f_{j}(a)=p^{-2} \Phi(a) \Phi\left(a^{p}\right)(1,0)+U_{j}
$$

for $p>2$ and $j=1, \ldots, p-2$.

The number of these groups $\mathbf{C r y s}\left(C_{p^{2}} ; M ; T\right)$ is equal to $2 p-3$.

Corollary 1. There exist at least $2 p-3$ Bieberbach groups (in the classical sense) with cyclic indecomposable holonomy group of order $p^{2}$.

Theorem 3. Let $G=\langle a\rangle \times\langle b\rangle \cong C_{2} \times C_{2}$ and let $K$ be one of the rings $\mathbb{Z}, \mathbb{Z}_{2}, \mathbb{Z}_{(2)}$. Let $F$ be a field containing $K$, let $M$ be a $K G$-module corresponding to the indecomposable K-representation $\Gamma$ of $G$, and let $f: G \rightarrow \hat{M}=F M^{+} / M^{+}$be a cocycle. The following table lists the choices of $\Gamma$ and $f$ which define, up to isomorphism, all torsion-free indecomposable groups $\mathbf{C r n s}(G ; M ; f)$.

\begin{tabular}{|c|c|c|c|c|}
\hline$N:$ & $m$ & $\Gamma$ & $f(a)=\left(x_{1}, \ldots, x_{m}\right)+M, f(b)=\left(y_{1}, \ldots, y_{m}\right)+M$ & $t_{m}$ \\
\hline 1 & $\begin{array}{c}4 n+1 \\
(n \geqslant 1)\end{array}$ & $\Delta_{n}$ & $\begin{array}{c}x_{n+1}=\frac{1}{2}, x_{i}=0(i \neq n+1), \\
y_{1}=\frac{1}{2}, 2 y_{2}=\cdots=2 y_{n+1}=0, \\
y_{2}+\cdots+y_{n+1}=\frac{1}{2}, \\
y_{n+2}=\cdots=y_{4 n+1}=0\end{array}$ & $2^{n-1}$ \\
\hline 2 & $\begin{array}{c}4 n+4 \\
(n \geqslant 0)\end{array}$ & $W_{n}^{*}$ & $\begin{array}{c}x_{2 n+3}=\frac{1}{2}, x_{i}=0(i \neq 2 n+3), \\
y_{1}=0, y_{2}=\frac{1}{2}, y_{3}=\cdots=y_{3 n+3}=0, \\
2 y_{3 n+4}=\cdots=2 y_{4 n+3}=0, y_{4 n+4}=\frac{1}{2}\end{array}$ & $2^{n}$ \\
\hline 3 & 5 & $\Delta_{1}^{*}$ & $f(a)=\left(0, \frac{1}{2}, 0,0,0\right), f(b)=\left(\frac{1}{2}, 0, \frac{1}{2}, \frac{1}{4}, 0\right)$ & 1 \\
\hline 4 & 8 & $W_{1}$ & $f(a)=\left(0,0,0,0, \frac{1}{2}, 0,0,0\right), f(b)=\left(0, \frac{1}{2}, 0, \frac{1}{4}, 0, \frac{1}{2}, 0, \frac{1}{2}\right)$ & 1 \\
\hline
\end{tabular}


Here, $m$ is the degree of the representation $\Gamma, f$ is the cocycle, and $t_{m}$ is the number of torsion-free groups.

\section{Preliminary results and Theorem 1}

Let $K=\mathbb{Z}, \mathbb{Z}_{(p)}$ or $\mathbb{Z}_{p}$ as above. We point out that in these cases the group $H^{1}(G, \hat{M})$ is finite. Denote by $C_{p^{n}}=\left\langle a \mid a^{p^{n}}=1\right\rangle$ the cyclic group of order $p^{n}$. The following three lemmas and Corollary 2 are well known and they can be found for example in $[1]$.

Lemma 1. Let $K$ be one of the rings $\mathbb{Z}, \mathbb{Z}_{p}, \mathbb{Z}_{(p)}$. For $i=1,2$, let $G_{i}$ be a finite group and $\Gamma_{i}, M_{i}, T_{i}$ be the representation, the module and the cocycle associated with $G_{i}$ as in the Introduction. The groups $\mathbf{C r y s}\left(G_{1} ; M_{1} ; T_{1}\right)$ and $\mathbf{C r y s}\left(G_{2} ; M_{2} ; T_{2}\right)$ are isomorphic if and only if there exist a group isomorphism $\varepsilon: G_{1} \rightarrow G_{2}$ and a K-module isomorphism $\tau: M_{1} \rightarrow M_{2}$ which satisfy the following conditions:

(1) $\varepsilon(g) \tau=\tau g$ in $M_{1}$, for all $g \in G_{1}$;

(2) the cocycles $T_{2}$ and $T_{1}^{\varepsilon}$ are cohomologous (here, $T_{1}^{\varepsilon}(g)=\tau^{\prime} T_{1}\left(\varepsilon^{-1} g\right)$ for all $g \in G_{2}$, where $\tau^{\prime}: \hat{M}_{1} \rightarrow \hat{M}_{2}$ is the homomorphism induced by $\left.\tau\right)$.

Lemma 2. Suppose that the character of the K-representation $\Gamma$ of $C_{n}$ does not contain the trivial character as a summand. Then $H^{1}\left(C_{n}, \hat{M}\right)$ is trivial.

Proof. Since 1 is not an eigenvalue of the operator $a$, which acts on $F M$, the operator $a-1$ is a unit. This means that $T(a)=(a-1) x+M$ for some $x \in F M$, i.e. $B^{1}\left(C_{n}, \hat{M}\right)=C^{1}\left(C_{n}, \hat{M}\right)$.

Lemma 3. Let $G \cong C_{p^{s}}$ and $M$ be a projective $K G$-module. Then $H^{1}\left(C_{p^{s}}, \hat{M}\right)$ is trivial.

Proof. Since some direct sum $M \oplus \cdots \oplus M$ of copies of $M$ is a free $K C_{p^{s}}$-module, it is sufficient to prove the lemma for $M=K C_{p^{s}}$. Let $T(a)=x+M$ where

$$
x=\lambda\left(1+a+\cdots+a^{p^{s}-1}\right)+u_{1}(a-1) \in F M,
$$

and where $\lambda \in F$ and $u_{1} \in F C_{p^{s}}$. From the condition $\left(1+a+\cdots+a^{p^{s}-1}\right) T(a) \subset M$ it follows that $\lambda p^{s} \in K$. Then $x-\lambda p^{s}=u_{2}(a-1)$, where $u_{2} \in F C_{p^{s}}$. Therefore $T$ is a coboundary.

Corollary 2. Suppose that the K-representation $\Gamma$ of $C_{p}$ does not contain the trivial $K$ representation as a summand. Then $H^{1}\left(C_{p}, \hat{M}\right)$ is trivial.

Proof. The $K$-representation $\Gamma$ of $C_{p}$ is a direct sum $\Gamma=\Gamma_{1} \oplus \Gamma_{2}$, where $\Gamma_{1}$ is a sum of copies of the irreducible $K$-representation of degree $p-1$ and $\Gamma_{2}$ is a $K$-representation corresponding to a projective $K C_{p}$-module. The proof follows by applying Lemma 1 to $\Gamma_{1}$ and Lemma 3 to $\Gamma_{2}$. 
For the proof of Theorem 1 we consider certain $K$-representations of the group $\langle a\rangle=C_{p^{s}}$. Let $\xi_{t}$ be a primitive $p^{t}$ th root of unity and set $\xi_{t-1}=\xi_{t}^{p}$ for $t \geqslant 1$. Put

$$
B_{0}=\{1\}, \quad B_{1}=\left\{1, \xi_{1}, \ldots, \xi_{1}^{p-2}\right\}, \quad B_{j}=\bigcup_{i=0}^{p-1} \xi_{j}^{i} B_{j-1} \quad(j \geqslant 2) .
$$

Thus for each $t \leqslant s$ the set $B_{t}$ is a $K$-basis of the ring $K_{t}=K\left[\xi_{t}\right]$, which is a $K C_{p^{s-}}$ module with action defined by $a(\alpha)=\xi_{t} \alpha$ for $\alpha \in K_{t}$. The set $B_{t}$ is also an $F$-basis of the space $F K_{t}$ for each $t$.

Let $\delta_{t}$ be the matrix $K$-representation of $C_{p^{s}}$ corresponding to the $K$-basis $B_{t}$ of the module $K_{t}$. We note that $\delta_{t}$ is an irreducible $K$-representation of $C_{p^{s}}$ and $\delta_{t}^{p}(a)=$ $E_{p} \otimes \delta_{t-1}(a)$, where $E_{p}$ is the $p \times p$ identity matrix. Let

$$
\Delta_{1}=\delta_{0}^{(n)}+\delta_{1}^{(n)}, \quad \Delta_{2}=\delta_{2}^{(n)}+\delta_{s}^{(n)}
$$

be sums of $2 n$ irreducible $K$-representations of $C_{p^{s}}$, where

$$
\delta_{i}^{(n)}=\underbrace{\delta_{i}+\cdots+\delta_{i}}_{n} .
$$

Consider the $K$-representation $\Delta$ of $C_{p^{s}}$ defined by

$$
\Delta(a)=\left(\begin{array}{cc}
\Delta_{1}(a) & U(a) \\
0 & \Delta_{2}(a)
\end{array}\right)
$$

where

$$
U(a)=\left(\begin{array}{ll}
E_{n} \otimes\langle 1\rangle_{0} & J_{n} \otimes\langle 1\rangle_{0} \\
E_{n} \otimes\langle 1\rangle_{1} & E_{n} \otimes\langle 1\rangle_{1}
\end{array}\right)
$$

Here, $J_{n}$ is the Jordan block of order $n$ with entries 1 on the main diagonal and $\langle\omega\rangle_{t}$ denotes the matrix with all columns zero except the last one, which consists of the coordinates of the element $\omega \in K_{t}$ written in the basis $B_{t}(t=0,1)$.

Lemma 4. (see $[2,3])$. The K-representation $\Delta$ of $C_{p^{s}}$ is indecomposable.

Lemma 5. Let $x \in F K_{t}$ (where $\left.t>0\right)$ and suppose that $(a-1) x \in K_{t}$. Then $p x \in K_{t}$ and all coordinates of the vector px are multiples of the last coordinate.

Proof. The $K$-basis $B_{t}$ in $K_{t}$ is an $F$-basis in $F K_{t}$. Consider the coordinates of the column vectors in $F K_{t}$ and the matrix $\delta_{t}(a)$ of the operator $a$ in this basis. The lemma is easily checked successively for $t=1,2, \ldots$.

Let $B$ be a $K$-basis of the $K$-module $M_{\Delta}$ affording the matrix $K$-representation $\Delta$ 
of $\langle a\rangle=C_{p^{s}}$. Denote the first basis element by $v$. It is easy to see that $B$ is an $F$-basis in $F M_{\Delta}$. We define the function

$$
T_{\Delta}: C_{p^{s}} \rightarrow \widehat{M_{\Delta}}=F M_{\Delta}^{+} / M_{\Delta}^{+},
$$

by setting $T_{\Delta}\left(a^{j}\right)=j p^{-s} v+M_{\Delta}$ for $j=0,1, \ldots, p^{s}-1$.

Lemma 6. The function $T_{\Delta}$ is a 1-cocycle of $C_{p^{s}}$ with values in $\widehat{M_{\Delta}}$, and it is not cohomologous to the zero cocycle at the element $b=a^{p^{s-1}}$ of order $p$.

Proof. The first assertion follows from the definition of $T_{\Delta}$. To prove the second assertion, consider the $p$ th power $\Delta^{p}(a)$ of $\Delta$. We note that

$$
\Delta^{p}(a)=\left(\begin{array}{cc}
\Delta_{1}^{p}(a) & U^{\prime}(a) \\
0 & \Delta_{2}^{p}(a)
\end{array}\right) \quad \text { and } \quad \Delta_{1}^{p}(a)=E .
$$

Clearly the first row in $U^{\prime}(a)$ has the form

$$
\left(\langle 1\rangle_{0}, \ldots,\langle 1\rangle_{0},\langle 1\rangle_{0}, \ldots,\langle 1\rangle_{0}\right),
$$

and the row of matrices corresponding to the first of the representations $\delta_{1}^{p}$ will take the form of the following matrix:

$$
\left(\langle 1\rangle_{1},\left\langle\xi_{1}\right\rangle_{1}, \ldots,\left\langle\xi_{1}^{p-1}\right\rangle_{1},\langle 1\rangle_{1}, \ldots,\left\langle\xi_{1}^{p-1}\right\rangle_{1}\right)
$$

Subtracting the rows of this matrix from the first row in $U^{\prime}(a)$, we obtain a row in which all the entries are multiples of $p$. This transformation of rows in $U^{\prime}(a)$ corresponds to the replacement of some basis elements $u \in B(u \neq v)$ by $u^{\prime}=u \pm v$. We carry out this replacement; let $\Delta^{\prime}$ be the $K$-representation of $C_{p^{s}}$ in the new $K$-basis of $M_{\Delta}$. It is easy to see that the change of basis does not change the values of the function $T_{\Delta}$.

Let $H=\left\langle b \mid b=a^{p^{s-1}}\right\rangle$ and let $\Delta_{H}^{\prime}$ be the restriction of the representation $\Delta^{\prime}$ to $H$. Then

$$
\Delta_{H}^{\prime}(b)=\left(\begin{array}{cc}
\delta_{0}^{\left(m_{1}\right)}(a) & U^{\prime \prime}(b) \\
0 & \delta_{1}^{\left(m_{2}\right)}(a)
\end{array}\right)
$$

where, as shown above, all entries of the first row in $U^{\prime \prime}(b)$ are multiples of $p$. Let $M_{\Delta}=M_{1} \oplus M_{2}$ be the decomposition of $M_{\Delta}$ as a direct sum corresponding to the representations $\delta_{0}^{\left(m_{1}\right)}$ and $\delta_{1}^{\left(m_{2}\right)}$.

Suppose that $T_{\Delta}$ is cohomologous to the trivial cocycle at $H$. Then there exists a vector $x \in F M_{\Delta}$ such that

$$
T_{\Delta}(b)=(b-1) x+M_{\Delta}
$$


Let $x=x_{1}+x_{2}$ with $x_{i} \in F M_{i}$ for $i=1,2$. Since the projection of $T_{\Delta}(b)$ on $F M_{2}$ is equal to zero (modulo $M_{\Delta}$ ), the projection of $(b-1) x=(b-1) x_{2}$ on $F M_{2}$ is also equal to zero. From Lemma 5 it follows that $p x_{2} \in M_{2}$. Let $\lambda$ be the coefficient of the basis vector $v$ in $(b-1) x$.

It is easy to see that $\lambda$ is a sum of products of the entries of the first row in $U^{\prime \prime}(b)$ (these entries are multiples of $p$ ) on the column which consists of coordinates of the vector $x_{2}$. Since $p x_{2} \in M_{2}$ it follows that $\lambda \in K$. Since $T_{\Delta}(b)=p^{-1} v+M_{\Delta}$, we have $\lambda=p^{-1}$. But $p^{-1} \notin K$, and so $\lambda \notin K$. This contradiction proves that $T_{\Delta}$ is not cohomologous to zero at $H$. The lemma is proved.

Proof of Theorem 1. Let us consider the group $\operatorname{Crns}\left(C_{p^{s}} ; M_{\Delta} ; T_{\Delta}\right)$. If this group has an element of prime order, then this order can only be $p$ and, moreover, the cocycle $T_{\Delta}$ must be cohomologous to the zero cocycle at the element $b=a^{p^{s-1}}$ in $C_{p^{s}}$. By Lemma 6 this is impossible. Therefore $\operatorname{Crns}\left(C_{p^{s}} ; M_{\Delta} ; T_{\Delta}\right)$ is torsion-free. Moreover this group is indecomposable (see Lemma 4).

\section{Theorem 2}

Now let $\langle a\rangle=C_{p^{2}}$. We want now to find all groups $\operatorname{Crys}\left(C_{p^{2}} ; M ; T\right)$ which are torsion-free. Put

$$
\Phi(x)=x^{p-1}+x^{p-2}+\cdots+x+1 .
$$

There exists a unit $\theta$ in $K C_{p^{2}}$ such that

$$
(a-1)^{p} \Phi\left(a^{p}\right)=p(a-1) \theta \Phi\left(a^{p}\right) .
$$

For $0 \leqslant i \leqslant p-2$, let $X_{i}$ be the $K C_{p^{2}}$-submodule of $K C_{p^{2}}$ generated by the following elements:

$$
u=\Phi(a) \Phi\left(a^{p}\right), \quad \omega=(a-1) \Phi\left(a^{p}\right), \quad v=\Phi(a)+(a-1)^{i+1} .
$$

It is easy to see that

$$
(a-1) u=0, \quad \Phi(a) \omega=0, \quad \Phi\left(a^{p}\right) v=u+(a-1)^{i} \omega .
$$

From these equations it follows that the $K$-representation $\Gamma_{i}$ of the group $C_{p^{2}}$ in the $K$-basis

$$
u ; \quad \omega, a \omega, a^{2} \omega, \ldots, a^{p-2} \omega ; \quad a^{l} v, a^{l+p} v, \ldots, a^{l+p(p-2)} v
$$

$(l=0,1, \ldots, p-1)$ corresponding to the module $X_{i}$, has the following form: 


$$
\Gamma_{i}(a)=\left(\begin{array}{ccc}
1 & 0 & \langle 1\rangle_{0} \\
& \delta_{1}(a) & \left\langle\alpha_{i}\right\rangle_{1} \\
& & \delta_{2}(a)
\end{array}\right)
$$

where $\alpha_{i}=\left(\xi_{1}-1\right)^{i}$ and $i=0,1, \ldots, p-2$.

Lemma 7. Let $H=\langle b\rangle$ where $b=a^{p}$. The $K H$-module $\left.X_{i}\right|_{H}$ is a direct sum of two $\mathrm{KH}$-submodules, one of which coincides with $\mathrm{Ku}$.

Proof. Consider the $K$-submodule $X_{i}^{\prime}$ in $X_{i}$ generated by the following $p^{2}-1$ elements of $X_{i}$ :

$$
\begin{gathered}
V=\left\{v, b v, \ldots, b^{p-2} v\right\}, \quad(a-1) V, \ldots,(a-1)^{p-2} V, \quad v^{\prime}=(a-1)^{p-1} v+\theta u, \\
b v^{\prime}, \ldots, b^{p-2} v^{\prime}, \quad \theta \omega_{1}, \ldots, \theta \omega_{i}, \quad u+\omega_{i+1}, \omega_{i+2}, \ldots, \omega_{p-1},
\end{gathered}
$$

where $\omega_{j}=(a-1)^{j} \Phi(b)=(a-1)^{j-1} \omega$ for $j=1, \ldots, p-1$.

Clearly $X_{i}$ is the direct sum of $K u$ and $X_{i}^{\prime}$. To prove the lemma it is sufficient to show that $X_{i}^{\prime}$ is a $K H$-module. We have

$$
\begin{array}{lll}
\Phi(b) v & =u+\omega_{i+1} \in X_{i}^{\prime}, \\
\Phi(b)(a-1) v & =\omega_{i+2} & \in X_{i}^{\prime}, \\
\ldots \ldots \ldots \ldots & \ldots \ldots & \ldots \\
\ldots \ldots \ldots & \ldots \ldots & \ldots \\
\Phi(b)(a-1)^{p-i-2} v & =\omega_{p-1} & \in X_{i}^{\prime}, \\
\Phi(b)(a-1)^{p-r-2+j} v=p \theta \omega_{j} & \in X_{r}^{\prime},
\end{array}
$$

for $0 \leqslant r \leqslant p-2, j=1, \ldots, r$ and

$$
\begin{aligned}
\Phi(b) v^{\prime} & =(a-1)^{p-1} \Phi(b) v+p \theta u=(a-1)^{p+i} \Phi(b)+p \theta u \\
& =p \theta(a-1)^{1+i} \Phi(b)+p \theta u=p \theta\left(\omega_{i+1}+u\right) \in X_{i}^{\prime}
\end{aligned}
$$

These equations show that $X_{i}^{\prime}$ is a $K H$-submodule of $X_{i}$.

For $i=0,1, \ldots, p-2$ we introduce the cocycle

$$
T_{i}: C_{p^{2}} \rightarrow \widehat{X_{i}}=F X_{i}^{+} / X_{i}^{+}
$$

defined by $T_{i}(a)=p^{-2} u+X_{i}$.

Lemma 8. For $i=0,1, \ldots, p-2$ the group $\operatorname{Crys}\left(C_{p^{2}} ; X_{i} ; T_{i}\right)$ is torsion-free. 
Proof. Since $T_{i}\left(a^{p}\right)=p^{-1} u+X_{i} \neq X_{i}$ it follows from Lemma 7 that

$$
\left(\left(a^{p}-1\right) F X_{i}+X_{i}\right) \cap\left(F u+X_{i}\right)=X_{i}
$$

These conditions show that the cocycle $T_{i}$ is not cohomologous to the zero cocycle at the element $a^{p}$. This means that $\mathfrak{C r y s}\left(C_{p^{2}} ; X_{i} ; T_{i}\right)$ is torsion-free.

For $i=0,1, \ldots, p-1$ let $Y_{i}$ be the $K C_{p^{2}}$-submodule $\left\langle\Phi(a),(a-1)^{i}\right\rangle$ of $K C_{p^{2}}$. The $K$-representation $\Gamma_{i}^{\prime}$ corresponding to $Y_{i}$ has the following form:

$$
\Gamma_{i}^{\prime}(a)=\left(\begin{array}{ccc}
1 & \langle 1\rangle_{0} & 0 \\
& \delta_{1}(a) & \left\langle\alpha_{i}\right\rangle_{1} \\
& & \delta_{2}(a)
\end{array}\right)
$$

where $\alpha_{i}=\left(\xi_{1}-1\right)^{i}$

Lemma 9. For each cocycle $T: C_{p^{2}} \rightarrow \hat{Y}_{i}=F Y_{i}^{+} / Y_{i}^{+}$the group Crys $\left(C_{p^{2}} ; Y_{i} ; T\right)$ has an element of order $p$.

Proof. It is easy to see that each cocycle of $C_{p^{2}}$ with a value in $\hat{Y}_{i}$ will be cohomologous to a cocycle $T$ such that $T(a)=\lambda p^{-2} u+Y_{i}$, where $\lambda \in K, u=\Phi(a) \Phi(b)$. Thus $T\left(a^{p}\right)=p T(a)=\lambda p^{-1} u+Y_{i}$, and so to prove the lemma it is sufficient to show that $p^{-1} u \in\left(a^{p}-1\right) F Y_{i}+Y_{i}$. It is easy to see that

$$
\left(a^{p-1}+a^{p-2}+\cdots+a+1\right)-(a-1)^{p-1}=p \omega_{1}(a)
$$

where $\omega_{1}(a) \in K C_{p_{i}^{2}}$.

Let $v_{1}=(a-1)^{i}$. Then from (2) it follows that

$$
\left(\Phi\left(a^{p}\right)-p\right)(a-1)^{p-i-1} v_{1}=u-p \omega_{1}(a) \Phi\left(a^{p}\right)-p(a-1)^{p-i-1} v=u+p y,
$$

where $y \in Y_{i}$. Since $\Phi\left(a^{p}\right)-p=\left(a^{p}-1\right) z$, where $z \in K C_{p^{2}}$, we have

$$
p^{-1} u+Y_{i}=\left(a^{p}-1\right) p^{-1} z+Y_{i}
$$

which completes the proof of the lemma.

Let $p \neq 2$. In the free $K C_{p^{2}}$-module

$$
\left(K C_{p^{2}}\right)^{(2)}=\left\{\left(x_{1}, x_{2}\right) \mid x_{1}, x_{2} \in K C_{p^{2}}\right\}
$$

we consider the $K C_{p^{2}}$-submodule

$$
U_{j}=\left\langle\left((a-1)^{j+1}+\Phi(a),(a-1)^{j}\right), \Phi\left(a^{p}\right)(a-1,1)\right\rangle,
$$


for $1 \leqslant j \leqslant p-2$. The $K$-representation of $C_{p^{2}}$ corresponding to $U_{j}$ has the form

$$
\Gamma_{j}^{\prime \prime}: a \mapsto\left(\begin{array}{cccc}
1 & 0 & 0 & \langle 1\rangle_{0} \\
& 1 & \langle 1\rangle_{0} & 0 \\
& & \delta_{1}(a) & \left\langle\alpha_{j}\right\rangle_{1} \\
& & & \delta_{2}(a)
\end{array}\right),
$$

where $\alpha_{j}=\left(\xi_{1}-1\right)^{j}$ and $j=1,2, \ldots, p-2$. Define the cocycle

$$
f_{j}: C_{p^{2}} \rightarrow \widehat{U_{j}}=F U_{j}^{+} / U_{j}^{+}
$$

by $f_{j}(a)=p^{-2} \Phi(a) \Phi\left(a^{p}\right)(1,0)+U_{j}$.

Lemma 10. For $j=1, \ldots, p-2$ the group $\mathfrak{C r y s}\left(C_{p^{2}} ; U_{j} ; f_{j}\right)$ is torsion-free.

Proof. Let $u_{1}=\Phi(a) \Phi\left(a^{p}\right)(1,0)$ and $u_{2}=\Phi(a) \Phi\left(a^{p}\right)(0,1)$. It is easy to see that the sequence of $K C_{p^{2}}$-modules

$$
0 \rightarrow K u_{2} \rightarrow U_{j} \rightarrow X_{j} \rightarrow 0
$$

is exact. The cocycle $f_{j}$ induces the cocycle $T_{j}: C_{p^{2}} \rightarrow \widehat{X_{j}}$ defined in (1), which is not equal to the zero cocycle on the group $H=\left\langle a^{p}\right\rangle$ by Lemma 8 . Therefore $f_{j}$ is also non-cohomologous to the zero cocycle in $H$. This means that $\mathfrak{C r y s}\left(C_{p^{2}} ; U_{j} ; f_{j}\right)$ has no elements of order $p$.

We consider one more $K C_{p^{2}}$-module, namely the submodule $U_{0}$ of $K C_{p^{2}}$ generated by $\Phi(a)$. The corresponding $K$-representation of $C_{p^{2}}$ has the form

$$
a \mapsto\left(\begin{array}{cc}
1 & \langle 1\rangle_{0} \\
0 & \delta_{2}(a)
\end{array}\right) .
$$

Lemma 11. For each cocycle $T: C_{p^{2}} \rightarrow U_{0}$ the group $\operatorname{Crys}\left(C_{p^{2}} ; U_{0} ; T\right)$ has an element of order $p$.

Proof. It is easy to see that any cocycle of $C_{p^{2}}$ with values in $\widehat{U_{0}}=F U_{0}^{+} / U_{0}^{+}$is cohomologous to a cocycle $T$ of the form

$$
T(a)=\lambda p^{-2} \Phi(a) \Phi\left(a^{p}\right)+U_{0}
$$

with $\lambda \in K$. Replacing $a$ by $a^{p}$ in (2) we have

$$
p^{-1} \Phi(a) \Phi\left(a^{p}\right)=p^{-1}\left(a^{p}-1\right)^{p-1} \Phi(a)+\omega_{1}\left(a^{p}\right) \Phi(a) .
$$

Then $T\left(a^{p}\right)=(a-1) z+U_{0}$, where $z \in F U_{0}$, and this proves the lemma. 
Proof of Theorem 2. From the description in [2] of the $K$-representations of $C_{p^{2}}$ it follows that the indecomposable $K C_{p^{2}}$-modules corresponding to the faithful $K$-representations of $C_{p^{2}}$ whose characters contain the trivial character are the following:

$$
\begin{gathered}
X_{i}(i=0,1, \ldots, p-2), \quad Y_{j}(j=0,1, \ldots, p-1), \\
U_{0}, \quad U_{k}(k=1, \ldots, p-2) .
\end{gathered}
$$

By Lemmas 9 and 11 we are interested only in the modules $X_{i}$ and $U_{j}$. Let us consider the module $X_{i}$ where $0 \leqslant i \leqslant p-2$. It is easy to see that Lemma 2 can be applied to the factor module $X_{i} / K v$, where $v=\Phi(a) \Phi\left(a^{p}\right)$. Therefore any cocycle of $C_{p^{2}}$ with the values in $\widehat{X_{i}}$ will be cohomologous to a cocycle $T$ of the form

$$
T(a)=\lambda p^{-2} v+X_{i}
$$

with $\lambda \in K$. We claim that if in this equation $\lambda \equiv 0(\bmod p)$ then $T$ is cohomologous to the trivial cocycle. From (2) we have

$$
p^{-1} \Phi(a) \Phi\left(a^{p}\right)+p^{-1} \Phi\left(a^{p}\right)(a-1)^{i+1}=p^{-1}\left(a^{p}-1\right)^{p-1} \theta_{i}+\omega_{1}\left(a^{p}\right) \theta_{i},
$$

where $\theta_{i}=\Phi(a)+(a-1)^{i+1} \in X_{i}$. We will use the equation

$$
\Phi\left(a^{p}\right)(a-1)^{p}=p(a-1) \Phi\left(a^{p}\right) \omega_{2},
$$

where $\omega_{2}$ is a unit in $K C_{p^{2}}$. From (6) it follows that

$$
p^{-1}(a-1)^{i+1} \Phi\left(a^{p}\right)=p^{-2} \Phi\left(a^{p}\right)(a-1)^{p+i} \omega_{2}^{-1} \in(a-1) F X_{i},
$$

for $i=0,1, \ldots, p-2$. Then from (6) one finds that

$$
p^{-1} \Phi(a) \Phi\left(a^{p}\right) \in(a-1) F X_{i}+X_{i}
$$

for $i=0,1, \ldots, p-2$. Our claim follows.

From the above it follows that $H^{1}\left(C_{p^{2}}, \widehat{X_{i}}\right)$ is cyclic of order $p$ and that all elements of this group can be represented by the cocycles $T$ defined in (5) with $\lambda=$ $0,1, \ldots, p-1$.

We will show that each non-zero cocycle $T$ defines up to isomorphism the group $\operatorname{Crys}\left(C_{p^{2}} ; X_{i} ; T_{i}\right)$.

Let $\varepsilon$ be an automorphism of the group $C_{p^{2}}$ and $X_{i}^{\varepsilon}$ be the $K C_{p^{2}}$-module $X_{i}$ twisted by this automorphism, i.e.

$$
X_{i}^{\varepsilon}=X_{i}, \quad a \cdot x=\varepsilon(a) x, \quad \text { for } x \in X_{i}
$$


It is not difficult to show the existence of an automorphism $\tau$ of the $K$-module $X_{i}$ such that $\varepsilon(a) \tau=\tau a$ in $X_{i}$ and $\tau(v)=v$, where $v=\Phi(a) \Phi\left(a^{p}\right)$.

Let $\varepsilon^{-1}(a)=a^{s}$, with $(s, p)=1$. Since we have $a T_{i}(a)=T_{i}(a)$ and $\tau^{\prime}(\bar{v})=\bar{v}$, where $\bar{v}=v+X_{i}$, we obtain

$$
T_{i}^{\varepsilon}(a)=\tau^{\prime} T_{i}\left(\varepsilon^{-1}(a)\right)=s T_{i}(a)=s p^{-2} v+X_{i} .
$$

From Lemma 1 it follows that $\mathfrak{C r y s}\left(C_{p^{2}} ; X_{i} ; T_{i}\right)$ is isomorphic to $\operatorname{Crys}\left(C_{p^{2}} ; X_{i} ; T\right)$, where $T(a)=s p^{-2} v+X_{i}$. We have shown that each group $\operatorname{Crys}\left(C_{p^{2}} ; X_{i} ; T\right)$ with $T \not \equiv 0$ is isomorphic to $\mathbb{C} \mathfrak{r y s}\left(C_{p^{2}} ; X_{i} ; T_{i}\right)$ for some $i$.

Now consider groups of the form $\operatorname{Crns}\left(C_{p^{2}} ; U_{j} ; T\right)$. First we remark that $H^{1}\left(C_{p^{2}}, \widehat{Y}_{j}\right)$ is cyclic of order $p$, for $j=1, \ldots, p-1$. The proof of this is similar to the proof for the group $H^{1}\left(C_{p^{2}}, \widehat{X_{i}}\right)$. Since $Y_{0}=K C_{p^{2}}$, we have $H^{1}\left(C_{p^{2}}, \widehat{Y_{0}}\right)=0$ (see Lemma 3).

Let $u_{1}, u_{2}, \ldots, u_{p^{2}+1}$ be a $K$-basis in $U_{j}$ such that

$$
u_{1}=\Phi(a) \Phi\left(a^{p}\right)(1,0) \quad \text { and } \quad u_{2}=\Phi(a) \Phi\left(a^{p}\right)(0,1)
$$

and for $0 \leqslant \alpha, \beta \leqslant p-1$ let the cocycle $T_{\alpha, \beta}$ satisfy

$$
T_{\alpha, \beta}(a)=p^{-2}\left(\alpha u_{1}+\beta u_{2}\right)+U_{j}
$$

We use the exact sequence (4) and the exact sequence

$$
0 \rightarrow K u_{1} \rightarrow U_{j} \rightarrow Y_{j} \rightarrow 0
$$

This enables us to show that any cocycle $T: C_{p^{2}} \rightarrow U_{j}$ is cohomologous to some cocycle $T_{\alpha, \beta}$ with $0 \leqslant \alpha, \beta \leqslant p-1$.

By Lemma 9 and (7), the cocycle $T_{0, \beta}$ is cohomologous to the zero cocycle at the element $a^{p}$ of $C_{p^{2}}$ and therefore $\mathfrak{C} \mathfrak{r y s}\left(C_{p^{2}} ; U_{j} ; T_{0, \beta}\right)$ has an element of order $p$.

Now let $\alpha \neq 0$. Then $\alpha$ is a unit in $K$ and the map $\tau$ defined by $\tau(x)=\alpha x$ is an automorphism of the $K C_{p^{2}}$-module $U_{j}$. It follows that the cocycle $T_{\alpha, \beta}(\alpha \neq 0)$ can be replaced by $T_{1, \alpha^{-1} \beta}$. So it is enough to consider the cocycles $T_{1, \beta}$, where $\beta=0$, $1, \ldots, p-1$. We will show that $\mathfrak{C} \mathfrak{r y s}\left(C_{p^{2}} ; U_{j} ; T_{1, \beta}\right)$ is isomorphic to $\operatorname{Crys}\left(C_{p^{2}} ; U_{j} ; f_{j}\right)$ (note that $f_{j}=T_{1,0}$ ).

We replace the basis element $u_{1}$ by $u_{1}^{\prime}=u_{1}+\beta u_{2}$ in $U_{j}$. Then

$$
T_{1, \beta}(a)=p^{-2} u_{1}^{\prime}+U_{j}
$$

Let $Y_{j}^{\prime}=U_{j} / K u_{1}^{\prime}$. Then the $K$-representation $\Gamma_{j}^{\prime \prime \prime}$ corresponding to $Y_{j}^{\prime}$ is

$$
\Gamma_{j}^{\prime \prime \prime}: a \rightarrow\left(\begin{array}{ccc}
1 & \langle 1\rangle_{0} & \langle-\beta\rangle_{0} \\
& \delta_{1}(a) & \left\langle\alpha_{j}\right\rangle_{1} \\
& & \delta_{2}(a)
\end{array}\right) .
$$


This representation is equivalent to $\Gamma_{j}^{\prime}$. Because of this equivalence we will replace the basis elements $u_{2}, \ldots, u_{p^{2}+1}$ by $u_{2}^{\prime}, \ldots, u_{p^{2}+1}^{\prime}$. Then in the $K$-basis $u_{1}^{\prime}, u_{2}^{\prime}, \ldots, u_{p^{2}+1}^{\prime}$ the operator $a$ has the same matrix (3) as in the basis $u_{1}, u_{2}, \ldots, u_{p^{2}+1}$. Define an automorphism $\tau: U_{j} \rightarrow U_{j}$ of the $K$-module $U_{j}$ by $\tau\left(u_{i}^{\prime}\right)=u_{i}$ for $i=1, \ldots, p^{2}+1$. We have $\tau a=a \tau$ and moreover

$$
\tau^{\prime} T_{1, \beta}(a)=\tau^{\prime}\left(p^{-2} u_{1}^{\prime}+U_{j}\right)=p^{-2} u_{1}+U_{j}=f_{j}(a)
$$

It follows from Lemma 1 that $\mathfrak{C} \mathfrak{r y s}\left(C_{p^{2}} ; U_{j} ; T_{1, \beta}\right)$ and $\mathfrak{C} \mathfrak{r y s}\left(C_{p^{2}} ; U_{j} ; f_{j}\right)$ are isomor-

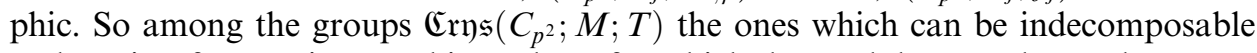
and torsion-free are isomorphic to those for which the module $M$ and cocycle $T$ were listed in this theorem. Now Lemmas 8 and 10 complete the proof.

\section{Theorem 3}

Let $G \cong C_{p} \times C_{p}$ with generators $a, b$ and let $K$ be one of the rings $\mathbb{Z}, \mathbb{Z}_{p}, \mathbb{Z}_{(p)}$. In the case when $p=2$ we will give a full description of the indecomposable torsion-free groups $\mathfrak{C r y s}\left(C_{2} \times C_{2} ; M ; T\right)$. We will use the classification of the indecomposable $K$ representations of $C_{2} \times C_{2}$ given by Nazarova in $[15,16]$.

Lemma 12. Let $M$ be the $K\left[C_{p} \times C_{p}\right]$-submodule of the free $K\left[C_{p} \times C_{p}\right]$-module $\left(K\left[C_{p} \times C_{p}\right]\right)^{(2)}$ defined as follows:

$$
M=\langle(\Phi(a), 0),(p, 0),(0, \Phi(b)),(0, p),(b-1,1-a)\rangle
$$

Then the following assertions hold:

(1) $M$ is an indecomposable $K\left[C_{p} \times C_{p}\right]$-module and $\operatorname{dim}_{K}(M)=2 p^{2}$;

(2) there exists a cocycle $T: C_{p} \times C_{p} \rightarrow \hat{M}=F M^{+} / M^{+}$defined by

$$
T(a)=(1,0)+M, \quad T(b)=(0,1)+M
$$

(3) the group $\mathfrak{C r y s}\left(C_{p} \times C_{p} ; M ; T\right)$ is torsion-free.

Proof. (1) Let $\overline{Z_{p}}=K / p K$ and regard $\overline{Z_{p}}$ as a $K\left[C_{p} \times C_{p}\right]$-module with $C_{p} \times C_{p}$ acting trivially. Consider the projective resolution

$$
\cdots \rightarrow\left(K\left[C_{p} \times C_{p}\right]\right)^{(3)} \stackrel{\tau_{1}}{\rightarrow}\left(K\left[C_{p} \times C_{p}\right]\right) \stackrel{\tau_{0}}{\rightarrow} \overline{Z_{p}} \rightarrow 0
$$

of $\overline{Z_{p}}$. It is easy to see that $\operatorname{ker}\left(\tau_{0}\right)=\langle a-1, b-1, p\rangle$, and

$$
\begin{aligned}
\operatorname{ker}\left(\tau_{1}\right)=\langle & (\Phi(a), 0,0),(0, \Phi(b), 0) \\
& (b-1,1-a, 0),(p, 0,1-a),(0, p, 1-b)\rangle .
\end{aligned}
$$


The $K\left[C_{p} \times C_{p}\right]$-modules $\operatorname{ker}\left(\tau_{0}\right)$ and $\operatorname{ker}\left(\tau_{1}\right)$ are indecomposable. Each $x \in \operatorname{ker}\left(\tau_{1}\right)$ has the form

$$
x=\left(u_{1} \Phi(a)+u_{3}(b-1)+p u_{4}, u_{2} \Phi(b)+u_{3}(1-a)+p u_{5}, u_{4}(1-a)+u_{5}(1-b)\right)
$$

with $u_{i} \in K\left[C_{p} \times C_{p}\right]$ for $i=1, \ldots, 5$. We map $x$ to the element

$$
\left(u_{1} \Phi(a)+u_{3}(b-1)+p u_{4}, u_{2} \Phi(b)+u_{3}(1-a)+p u_{5}\right)
$$

of $M$. It is easy to check that this defines an isomorphism of the $K\left[C_{p} \times C_{p}\right]$-modules $\operatorname{ker}\left(\tau_{1}\right)$ and $M$. Thus $M$ is an indecomposable $K\left[C_{p} \times C_{p}\right]$-module. Since $\operatorname{dim}_{K}\left(T_{0}\right)=$ $p^{2}$, we have

$$
\operatorname{dim}_{K}(M)=\operatorname{dim}_{K}\left(\operatorname{ker}\left(\tau_{1}\right)\right)=\operatorname{dim}_{K}\left(K\left[C_{p} \times C_{p}\right]\right)^{(3)}-\operatorname{dim}_{K}\left(\operatorname{ker}\left(\tau_{0}\right)\right)=2 p^{2} .
$$

(2) Define $T: C_{p} \times C_{p} \rightarrow \hat{M}$ as follows:

$$
\begin{gathered}
T\left(a^{i}\right)=\left(1+a+\cdots+a^{i-1}, 0\right)+M, \\
T\left(b^{j}\right)=\left(0,1+b+\cdots+b^{j-1}\right)+M, \\
T\left(a^{i} b^{j}\right)=a^{i} T\left(b^{j}\right)+T\left(a^{i}\right)+M ; \quad T(1)=M,
\end{gathered}
$$

for $0<i, j \leqslant p-2$. It is easy to see that $\Phi(a) T(a) \subset M, \Phi(b) T(b) \subset M$ and $(a-1) T(b)-(b-1) T(b) \subset M$. It follows that $T$ is a cocycle of $C_{p} \times C_{p}$ with values in $\hat{M}=F M^{+} / M^{+}$.

(3) It is sufficient to show that $T$ is not cohomologous to the zero cocycle at every non-trivial element $g$ of $C_{p} \times C_{p}$. Let $g=a^{i} b^{j}$, where $0<i, j \leqslant p-1$. Suppose that there exists $z \in F M$ such that

$$
T(g)=(g-1) z+M
$$

From the definition of $T$ and from (10) it follows that

$$
\left(1+a+\cdots+a^{i-1}, a^{i}\left(1+b+\cdots+b^{j-1}\right)\right)=(g-1) z+x
$$

for some $x \in M$. Multiplying this equation by $\Phi(a) \Phi(b)$ taking into account that

$$
\Phi(a) \Phi(b) M=p \Phi(a) \Phi(b)(K, K), \quad \text { and } \quad \Phi(a) \Phi(b)(g-1)=0
$$

we conclude that $(i, j) \in(p K, p K)$, which is impossible since $0<i, j \leqslant p-1$. This contradicts the assumption that $T$ is cohomologous to the zero cocycle at $g$ (see (10)).

Similarly, we may show that $T$ is not cohomologous to the zero cocycle at the remaining non-trivial elements of $C_{p} \times C_{p}$. Thus $\mathbb{C r y s}\left(C_{p} \times C_{p} ; M ; f\right)$ is torsion-free. 
Now let $p=2$, let $G=\langle a\rangle \times\langle b\rangle \cong C_{2} \times C_{2}$ and let $K$ be one of the rings $\mathbb{Z}, \mathbb{Z}_{2}$, $\mathbb{Z}_{(2)}$. We will study those groups $\mathfrak{C r y s}(G ; M ; T)$ which are torsion-free.

The group $G$ has the following irreducible $K$-representations:

$$
\begin{aligned}
\chi_{0}: a \mapsto 1, b \mapsto 1 ; & \chi_{1}: a \mapsto-1, b \mapsto 1 ; \\
\chi_{2}: a \mapsto-1, b \mapsto-1 ; & \chi_{3}: a \mapsto 1, b \mapsto-1 .
\end{aligned}
$$

Let $H=\langle h\rangle$ be a subgroup of $G$ of order 2. The indecomposable $K$-representations of $H$, up to equivalence, are the following:

$$
\gamma_{0}: h \mapsto 1 ; \quad \gamma_{1}: h \mapsto-1 ; \quad \gamma_{2}: h \rightarrow\left(\begin{array}{cc}
1 & 1 \\
0 & -1
\end{array}\right)
$$

Let $\Gamma$ be a $K$-representation of $G$ and $\left.\Gamma\right|_{H}$ its restriction to $H$. Let $M$ be a $K G$-module corresponding to the $K$-representation $\Gamma$ and $T: G \rightarrow \hat{M}$ be an arbitrary cocycle of $G$ with values in $\hat{M}=F M^{+} / M^{+}$(where $F$ is a field containing $K$ ). The following lemma gives necessary conditions for $\mathfrak{C r y s}(G ; M ; T)$ to be torsion-free.

Lemma 13. If $\operatorname{Crys}(G ; M ; T)$ is torsion-free then for each non-trivial subgroup $H$ of order 2 , the trivial representation $\gamma_{0}$ is contained in the decomposition of $\left.\Gamma\right|_{H}$ as a direct sum of indecomposable K-representations of $H$.

Indirect proof. Assume that $H$ is a subgroup of order 2 in $G$ such that $\left.\Gamma\right|_{H}$ does not have $\gamma_{0}$ as a direct summand. Then it follows from Lemmas 2 and 3 that any cocycle $T: G \rightarrow \hat{M}$ will be cohomologous in $H$ to the zero cocycle, and this implies that $\operatorname{Crys}(G ; M ; T)$ has elements of order 2 .

We make some remarks about the $K$-representations of $G \cong C_{2} \times C_{2}$. Let $G$ act trivially on $K$ and consider the projective resolution

$$
\begin{aligned}
\cdots & \rightarrow(K G)^{(n)} \stackrel{v_{n}}{\rightarrow}(K G)^{(n-1)} \rightarrow \cdots \\
& \quad \cdots \stackrel{v_{3}}{\rightarrow}(K G)^{(2)} \stackrel{v_{2}}{\rightarrow}(K G) \stackrel{v_{1}}{\rightarrow} K \rightarrow 0
\end{aligned}
$$

of $K$. Each $v_{n}$ is a homomorphism of the $K G$-modules and $\operatorname{ker}\left(v_{n}\right)$ is an indecomposable $K G$-module with

$$
\operatorname{dim}_{K}\left(\operatorname{ker}\left(v_{n}\right)\right)=2 n+1
$$

Let $\Gamma_{n}$ be the $K$-representation of $G$ corresponding to some $K$-basis in $\operatorname{ker}\left(v_{n}\right)$, and let $\Gamma_{n}^{*}$ be the contragradient $K$-representation of $\Gamma_{n}$, that is, $\Gamma_{n}^{*}(g)=\Gamma^{T}\left(g^{-1}\right)$ for all $g \in G$, where the superscript $T$ denotes transposition of matrices.

Lemma 14. (see $[16,22]$ ). Each indecomposable K-representation of $G \cong C_{2} \times C_{2}$ 
of odd degree is equivalent to just one of the following: $\chi_{i}, \Gamma_{n} \otimes_{K} \chi_{i}$ or $\Gamma_{n}^{*} \otimes_{K} \chi_{i}$, for some $i \in\{0,1,2,3\}$ and $n \geqslant 1$.

Let $p=2$ in (8) and let us consider the projective resolution for $\operatorname{ker}\left(\tau_{0}\right)=$ $\langle a-1, b-1,2\rangle$ :

$$
\begin{aligned}
& \cdots \rightarrow(K G)^{\left(t_{n}\right)} \stackrel{\tau_{n}}{\rightarrow}(K G)^{\left(t_{n-1}\right)} \rightarrow \cdots
\end{aligned}
$$

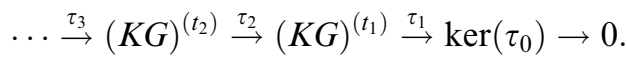

It is easy to show that in (13) we have $t_{n}=2 n+1$ and

$$
\operatorname{dim}_{K}\left(\operatorname{ker}\left(\tau_{n}\right)\right)=4 n+4
$$

where $n \geqslant 0$. Moreover all of the $K G$-modules $\operatorname{ker}\left(v_{n}\right)$ are indecomposable. If we take the tensor product over $K$ of the exact sequence (12) and the $K G$-module $\operatorname{ker}\left(\tau_{0}\right)$ and compare the result with the sequence (13), then we obtain easily the isomorphism

$$
\operatorname{ker}\left(\tau_{0}\right) \otimes_{K} \operatorname{ker}\left(v_{n}\right) \cong \operatorname{ker}\left(\tau_{n}\right) \oplus P_{n},
$$

where $P_{n}$ is a projective $K G$-module.

Lemma 15. Let $W_{n}$ be the K-representation of $G=\langle a\rangle \times\langle b\rangle \cong C_{2} \times C_{2}$ corresponding to the module $\operatorname{ker}\left(\tau_{n}\right)$ where $n \geqslant 0$. This representation has the following form:

$$
\begin{aligned}
& W_{0}: a \mapsto\left(\begin{array}{cccc}
1 & 1 & 0 & 1 \\
& -1 & 0 & 0 \\
& & 1 & 0 \\
& & & -1
\end{array}\right), \quad b \mapsto\left(\begin{array}{cccc}
1 & 1 & 1 & 0 \\
& -1 & 0 & 0 \\
& & -1 & 0 \\
& & & 1
\end{array}\right) \\
& W_{n}: a \mapsto\left(\begin{array}{ccccc}
D & 0 & 0 & 0 & 0 \\
& E_{n} & 0 & 0 & V_{n} \\
& & -E_{n} & V_{n} & 0 \\
& & & E_{n+1} & 0 \\
& & & & -E_{n+1}
\end{array}\right), \quad b \mapsto\left(\begin{array}{ccccc}
D & 0 & 0 & S & 0 \\
& E_{n} & 0 & V_{n}^{\prime} & 0 \\
& & -E_{n} & 0 & V_{n}^{\prime} \\
& & & -E_{n+1} & 0 \\
& & & & E_{n+1}
\end{array}\right) \text {, }
\end{aligned}
$$

where

$$
D=\left(\begin{array}{cc}
1 & 1 \\
0 & -1
\end{array}\right), \quad S=\left(\begin{array}{ccccc}
0 & \cdots & 0 & 1 & 1 \\
0 & \cdots & 0 & 0 & 0
\end{array}\right)
$$

and $V_{n}=\left(\begin{array}{ll}0 & E_{n}\end{array}\right), V_{n}^{\prime}=\left(E_{n} 0\right)$ are matrices with $n$ rows and $n+1$ columns for $n \geqslant 1$. 
Proof. The proof reduces to the determination of a $K$-basis of $\operatorname{ker}\left(\tau_{n}\right)$, and this is not difficult to construct by induction on $n$.

Lemma 16. Each faithful indecomposable K-representation of $G=\langle a\rangle \times\langle b\rangle$ which satisfies the necessary condition for the existence of a torsion-free group $\operatorname{Crys}(G ; M ; f)$ is one of the following:

$$
\Delta_{n}(n \geqslant 1) ; \quad \Delta_{n}^{*}(n \geqslant 1) ; \quad W_{n}(n \geqslant 0) ; \quad W_{n}^{*}(n \geqslant 0) .
$$

Here

$$
\Delta_{n}(a)=\left(\begin{array}{ccccc}
E_{n} & 0 & 0 & E_{n} & 0 \\
& 1 & 0 & 0 & 0 \\
& & -E_{n} & 0 & E_{n} \\
& & & -E_{n} & 0 \\
& & & & E_{n}
\end{array}\right), \quad \Delta_{n}(b)=\left(\begin{array}{ccccc}
1 & 0 & 0 & 0 & 0 \\
& E_{n} & 0 & 0 & E_{n} \\
& & -E_{n} & E_{n} & 0 \\
& & E_{n} & 0 \\
& & & & -E_{n}
\end{array}\right),
$$

and $\Delta_{n}^{*}$ and $W_{n}^{*}$ are $K$-representations of $G$ contragradient to $\Delta_{n}$ and $W_{n}$, so that $\Delta_{n}^{*}(g)=\Delta_{n}^{T}(g)$ and $W_{n}^{*}(g)=W_{n}^{T}(g)$ for all $g \in G$.

Proof. All $K$-representations listed above satisfy the necessary condition for the existence of a torsion-free group $\mathfrak{C r y s}(G ; M ; f)$. The analysis of all representations of odd degree (see Lemma 14) shows that among the representations $\Gamma_{n} \otimes \chi_{i}$ the necessary condition is satisfied only by $\Delta^{*}$ which is equivalent to $\Gamma_{2 n}(n=1,2, \ldots)$. Besides the representations $W_{n}$ and $W_{n}^{*}$, the group $G$ has a parameterized series of representations whose degrees are divisible by 4 . In this series the following pairs of matrices correspond to the pair of generating elements of $G$ :

$$
\left(\begin{array}{cccc}
E_{n} & 0 & 0 & E_{n} \\
& -E_{n} & E_{n} & 0 \\
& & E_{n} & 0 \\
& & & -E_{n}
\end{array}\right), \quad\left(\begin{array}{cccc}
E_{n} & 0 & \mathfrak{F} & 0 \\
& -E_{n} & 0 & E_{n} \\
& & -E_{n} & 0 \\
& & & E_{n}
\end{array}\right)
$$

where the matrix $\mathfrak{F}$ over $K$ has Frobenius (i.e. rational) canonical normal form indecomposable modulo $2 K$. Clearly the representations in this series do not satisfy the necessary condition. Consider the following pair of matrices:

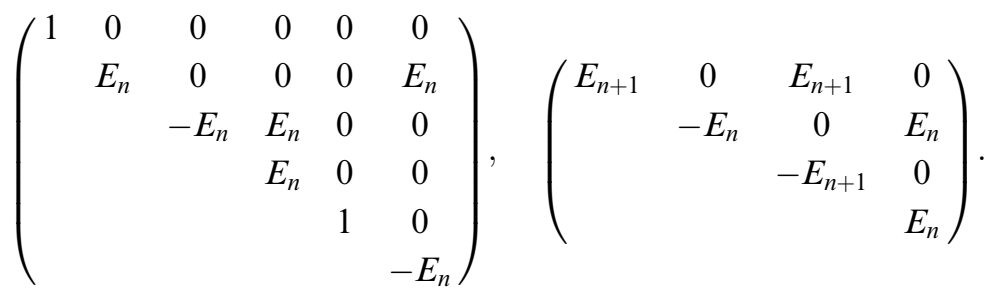


These matrices define indecomposable $K$-representations of $G$ of degree congruent to 2 modulo 4 and obviously these representations do not satisfy the necessary condition. We can obtain the remaining representations of degree $4 n-2$ either by the described process of tensor multiplication by irreducible $K$-representations or by taking contragradient representations.

As a result we get representations which do not satisfy the necessary condition for the existence of a torsion-free group $\mathfrak{C r y s}(G ; M ; f)$. Thus we have considered all indecomposable $K$-representations of $G$. The lemma is proved.

Proof of Theorem 3. We can take the module $M$ of a $K$-representation $\Gamma$ of $G$ of degree $m$ to be the $K$-module of $m$-dimensional columns with entries from $K$. Then $F M$ is the space of $m$-dimensional columns over $F$ and $\hat{M}=F M^{+} / M^{+}$is the group of $m$-dimensional columns with entries from $\hat{F}=F^{+} / K^{+}$. Let $f: G \rightarrow \hat{M}$ be a cocycle. The value $f(g)$ of $f$ at $g \in G$ is an $m$-dimensional column over $\hat{F}$. We note that if $g, h \in G$ then the product $g . f(h)$ is the ordinary product of the matrices $\Gamma(g)$ and $f(h)$.

If we consider the coordinates of the vector $f(g)$ as elements of $F$, then the elements of the ring $K$ will be replaced by 0 .

Let $\Gamma$ be any of the representations of $G$ listed in Lemma 16, let $M$ be the module of this representation and let $H=\langle h\rangle$ be a non-trivial subgroup of $G$. There exists only one basis vector $v$ in $M$ such that $M$ is the direct sum $M=K v \oplus M^{\prime}$ of the $K H$ module $K v$ and the $K H$-module $M^{\prime}$ generated by the rest of the basis vectors of $M$. In addition, $h v=v$ and a $K$-representation $\Gamma^{\prime}$ of $H$ corresponding to the module $M^{\prime}$ is a sum of representations of type $\gamma_{1}$ and $\gamma_{2}$ (see (11)). This allows us to replace the cocycle $f$ by a cohomologous cocycle $f_{1}$ in such a way that the projection $\left.f_{1}\right|_{\widehat{M}}$, will be equal to zero for the element $h$ (see Lemmas 2,3).

The coordinate $x_{v}$ of the vector $f(h)$ corresponding to the basis vector $v$ will be called the special component of the vector $f(h)$. From $(1+h) f(h)=0$, it follows that $2 x_{v}=0$ (in the group $\hat{F}$ ). For any $z \in \hat{M}$ the special component of $(h-1) z+f(h)$ is always equal to $x_{v}$. If $x_{v}=\frac{1}{2}$, then the cocycle $f$ is not cohomologous to the zero cocycle at $h$.

These remarks justify the following plan for the construction of cocycles of the representations $\Gamma$ from Lemma 16. The form of the representation $\Gamma$ defines the special components of the vectors $f(a)$ and $f(b)$ (where $a$ and $b$ are the generators of $G$ ). We choose $f(a)$ such that we can deduce that the special component is $\frac{1}{2}$ and all other components are zero. The possible forms of the components of the vector $f(b)$ follow from the following conditions:

$$
\begin{gathered}
(1+b) f(b)=0 ; \\
(a-1) f(b)=(b-1) f(a) .
\end{gathered}
$$

We will carry out the following operations on the vector $f(b)$ : replace $f(b)$ by the vector

$$
(b-1) z+f(b),
$$

where $z \in \hat{M}$ and $(a-1) z=0$. 
We discard all those forms of $f(b)$ with a zero special component. For a vector $f(b)$ whose special component equals $\frac{1}{2}$, we find that

$$
f(a b)=a f(b)+f(a)
$$

and we examine the solvability of the following equation

$$
(a b-1) z+f(a b)=0
$$

with $z \in \hat{M}$. The group $\operatorname{Crys}(G ; M ; f)$ is torsion-free if and only if the equation (17) has no solution.

We consider the following seven cases:

Case 1. Let $\Gamma=\Delta_{n}$. The special components are the $(n+1)$ th entry in $f(a)$ and the first one in $f(b)$. Set the $(n+1)$ th coordinate of $f(a)$ to $\frac{1}{2}$ and let all the rest be 0 . Let

$$
f^{T}(b)=\left(y, Y_{1}, Y_{2}, Y_{3}, Y_{4}\right)
$$

where $y \in \hat{F}, Y_{i} \in \hat{F}^{(n)}$ and $i=1,2,3,4$.

Using the operation (16) we can replace $Y_{2}$ by the zero vector. From (15) it follows that $Y_{3}=Y_{4}=0$, and from (14), it follows that $2 y=0$ and $2 Y_{1}=0$. Let $y=\frac{1}{2}, Y_{1}=$ $\left(v_{1}, v_{2}, \ldots, v_{n}\right)$. Using (17), it is easy to transform (18) to a linear system of equations (over $\hat{F}$ ) with the $(n+1) \times n$-matrix

$$
\left(\begin{array}{ccccc}
1 & 0 & \cdots & 0 & 0 \\
-1 & 1 & \ldots & 0 & 0 \\
\cdots & \cdots & \ldots & \cdots & \cdots \\
\cdots & \cdots & \cdots & \cdots & \cdots \\
0 & 0 & \cdots & -1 & 1 \\
0 & 0 & \cdots & 0 & -1
\end{array}\right)
$$

and coefficients $\frac{1}{2}, v_{1}, \ldots, v_{n-1}, v_{n}+\frac{1}{2}$. This system is solvable if and only if

$$
v_{1}+\cdots+v_{n-1}+v_{n}=0 .
$$

Case 2. Let $\Gamma=\Delta_{n}^{*}$. The matrices of the $K$-representation are the transposes of the matrices of $\Delta_{n}$. The special components of $f(a)$ and $f(b)$ are the same as in Case 1. Let us assume that $f(a)$ and $f(b)$ are chosen at first in the same way as in the case of $\Gamma=\Delta_{n}$ (see (19)). Condition (14) and operation (16) transform the vector $f(b)$ to the following form:

$$
f^{T}(b)=\left(y, 0,-2 Y_{3}, Y_{3}, 0\right) .
$$


Let $Y_{3}=\left(v_{1}, \ldots, v_{n-1}, v_{n}\right)$. It follows from (15) that if $n \geqslant 2$ then

$$
\begin{gathered}
y-2 v_{1}=0 ; \quad 2 v_{2}=\cdots=2 v_{n}=0 \\
2 v_{1}=0 ; \ldots ; 2 v_{n-1}=0 ; \quad 2 v_{n}=\frac{1}{2}
\end{gathered}
$$

and, if $n=1$, then $y-2 v_{1}=0,2 v_{1}=\frac{1}{2}$.

If $n>1$ and $y=\frac{1}{2}$, then (19) leads to a contradiction. If $n=1$ and $y=\frac{1}{2}$, then $v_{1}=\frac{1}{4}$.

Thus if $n>1$ and $f$ is a cocycle then the special component of the vector $f(b)$ is equal to zero. Then the cocycle $f$ is cohomologous to the zero cocycle in the element $b \in G$. This means that $\operatorname{Crys}(G ; M ; f)$ cannot be torsion-free if $M$ corresponds to the representation $\Gamma=\Delta_{n}^{*}$ where $n>1$.

Let $n=1$. Then

$$
f(a)=\left(0, \frac{1}{2}, 0,0,0\right), \quad f(b)=\left(\frac{1}{2}, 0, \frac{1}{2}, \frac{1}{4}, 0\right), \quad f(a b)=\left(\frac{1}{2}, 0, \frac{1}{2}, \frac{1}{4}, \frac{1}{2}\right) .
$$

It is easy to check that (18) is unsolvable.

Case 3. Let $\Gamma=W_{n}^{*}(n>0)$. The special components are the $(2 n+3)$ rd of $f(a)$ and the last of $f(b)$. Let the special component of $f(a)$ be equal to $\frac{1}{2}$, and let all the rest be zero.

Let $f^{T}(b)=\left(Y_{0}, Y_{1}, Y_{2}, Y_{3}, Y_{4}\right)$, where $Y_{0} \in \hat{F}^{(2)}, Y_{1}, Y_{2} \in \hat{F}^{(n)}, Y_{3}, Y_{4} \in \hat{F}^{(n+1)}$. Operation (16) allows us to replace $Y_{3}$ by the zero vector. It follows from (14) that $Y_{1}=0$. Condition (15) shows that $Y_{2}=0, Y_{0}=(0, y)(y \in \hat{F}, 2 y=0)$ and $2 Y_{4}=0$. Consequently

$$
f^{T}(b)=\left(0, y, 0, \ldots, 0, v_{1}, \ldots, v_{n}, \frac{1}{2}\right)
$$

The special component of $f(a b)$ is the second coordinate which, according to (17), equals $y$. Therefore $y=\frac{1}{2}$ and for any $v_{1}, \ldots, v_{n}\left(2 v_{1}=2 v_{2}=\cdots=2 v_{n}=0\right)$ the group $\operatorname{Crys}(G ; M ; f)$ is torsion-free.

Case 4. Let $\Gamma=W_{0}^{*}$. In this case it is easy to see that the cocycle $f$ with

$$
f(a)=\left(0,0, \frac{1}{2}, 0\right), \quad f(b)=\left(0, \frac{1}{2}, 0, \frac{1}{2}\right)
$$

determines a torsion-free group $\mathfrak{C r y s}(G ; M ; f)$.

Case 5. Let $\Gamma=W_{n}(n>1)$. We take the vectors $f(a)$ and $f(b)$ in the same fashion as in Case 3. Condition (14) shows that all components of the vector $Y_{4}$, except the last, are zero. Then condition (15) leads to a contradiction.

We obtain a contradiction by setting the special component in $f(a)$ equal to $\frac{1}{2}$. Consequently, for $\Gamma=W_{n}$ with $n>1$, any cocycle $f$ is cohomologous to the zero cocycle at the generator $a$ of $G$, and so $\operatorname{Crns}(G ; M ; T)$ is not torsion-free in this case. 
Case 6. Let $\Gamma=W_{1}$. For the cocycle $f$ with

$$
f(a)=\left(0,0,0,0, \frac{1}{2}, 0,0,0\right), \quad f(b)=\left(0, \frac{1}{2}, 0, \frac{1}{4}, 0, \frac{1}{2}, 0, \frac{1}{2}\right)
$$

the special components of the vector $f(a)$ (the fifth one) and $f(b)$ (the last one), and $f(a b)$ (the second one) are all equal to $\frac{1}{2}$. The cocycle $f$ determines a torsion-free group $\operatorname{Crys}(G ; M ; f)$ (see also Lemma 12).

Case 7. Let $\Gamma=W_{0}$. The special components are the third for $f(a)$ and the fourth for $f(b)$. Let us assume that they are equal to $\frac{1}{2}$. Then there exists only one cocycle

$$
f(a)=\left(0,0, \frac{1}{2}, 0\right), \quad f(b)=\left(0,0,0, \frac{1}{2}\right) .
$$

Hence $f(a b)=\left(\frac{1}{2}, 0, \frac{1}{2}, \frac{1}{2}\right)$ and the special component (the second one) for $f(a b)$ is equal to zero. The cocycle $f$ is cohomologous to zero at the element $a b$ and $\operatorname{Crys}(G ; M ; f)$ has elements of order 2 .

It follows from Lemma 16 that all $K$-representations $\Gamma$ of $G$ for which there are torsion-free groups $\operatorname{Crys}(G ; M ; f)$ have been enumerated. Consequently Theorem 3 is proved.

\section{References}

[1] D. J. Benson. Representations and cohomology, vol. 2. Cohomology of groups and modules. Cambridge Studies in Advanced Math. 31 (Cambridge University Press, 1998).

[2] S. D. Berman and P. M. Gudivok. Integral representations of finite groups. Soviet Math. Dokl. 3 (1962), 1172-1174.

[3] S. D. Berman and P. M. Gudivok. Indecomposable representations of finite group over the ring of $p$-adic integers. Izv. Akad. Nauk SSSR 28 (1964), 875-910.

[4] L. S. Charlap. Bieberbach groups and flat manifolds (Springer-Verlag, 1986).

[5] P. V. Z. Cobb. Manifolds with holonomy group $\mathbb{Z}_{2} \oplus \mathbb{Z}_{2}$ and the first Betti number zero. J. Differential Geometry 10 (1975), 221-224.

[6] C. W. Curtis and I. Reiner. Methods of representation theory, vol. 1. With applications to finite groups and orders (John Wiley \& Sons, Inc., 1981).

[7] P. M. Gudivok. Representations of finite groups over a complete discrete valuation ring. Algebra, number theory and their applications. Trudy Mat. Inst. Steklov 148 (1978), 96105.

[8] P. M. Gudivok and I. V. Shapochka. On the wildness of the problem of description of some classes of groups. Uzhgorod State Univ. Sci. Herald. Math. Ser. 3 (1998), 69-77.

[9] N. Gupta and S. Sidki. The group transfer theorem. Arch. Math. (Basel) 64 (1995), 5-7.

[10] N. Gupta and S. Sidki. On torsion free metabelian groups with commutator quotients of prime exponent. Internat J. Algebra Comput. 9 (1999), 493-520.

[11] A. Heller and I. Reiner. Representations of cyclic groups in rings of integers I. Ann. of Math. (2) 76 (1962), 73-92.

[12] A. Heller and I. Reiner. Representations of cyclic groups in rings of integers II. Ann. of Math. (2) 77 (1963), 318-328.

[13] G. Hiss and A. Szczepánski. On torsion-free crystallographic groups. J. Pure Appl. Algebra 74 (1991), 39-56. 
[14] G. M. Kopcha and V. P. Rudko. About torsion-free crystallographic group with indecomposable point cyclic p-group. Uzhgorod State Univ. Sci. Herald. Math. Ser. 3 (1998), $117-123$.

[15] L. A. Nazarova. Unimodular representations of the four group. Dokl. Akad. Nauk SSSR 140 (1961), 1011-1014.

[16] L. A. Nazarova. Representations of a tetrad. Izv. Akad. Nauk SSSR Ser. Mat 31 (1967), 1361-1378.

[17] W. Plesken. Kristallographische Gruppen. In Group theory, algebra, and number theory (Saarbrücken, 1993) (de Gruyter, 1996), pp. 75-96.

[18] W. Plesken. Some applications of representation theory. Prog. Math. 95 (1991), 477-496.

[19] J. P. Rossetti and P. A. Tirao. Compact flat manifolds with holonomy group $\mathbb{Z}_{2} \oplus \mathbb{Z}_{2}$. Proc. Amer. Math. Soc. 124 (1996), 2491-2499.

[20] J. P. Rossetti and P. A. Tirao. Five-dimensional Bieberbach groups with holonomy group $\mathbb{Z}_{2} \oplus \mathbb{Z}_{2}$. Geom. Dedicata 77 (1999), 149-172.

[21] J. P. Rossetti and P. A. Tirao. Compact flat manifolds with holonomy group $\mathbb{Z}_{2} \oplus \mathbb{Z}_{2}$, II. Rend. Sem. Mat. Univ. Padova 101 (1999), 99-136.

[22] V. P. Rudko. Algebras of integral p-adic representations of finite groups. Dokl. Akad. Nauk Ukrain. SSR Ser. A 11 (1979), 904-906.

Received 15 January, 2001; revised 11 June, 2001

V. A. Bovdi, Institute of Mathematics and Informatics, University of Debrecen, H-4010 Debrecen, P.O. Box 12, Hungary

E-mail: vbovdi@math.klte.hu

P. M. Gudivok, V. P. Rudko, Department of Algebra, Uzhgorod University, 88 000, Uzhgorod, Ukraine

E-mail: math1@univ.uzhgorod.ua 